\title{
Adult-onset hyperinsulinaemic hypoglycaemia in clinical practice: diagnosis, aetiology and management
}

\author{
Benjamin G Challis 1,2,3, Andrew S Powlson 1,2, Ruth T Casey2, Carla Pearson², \\ Brian Y Lam', Marcella Ma1, Deborah Pitfield², Giles S H Yeo', Edmund Godfrey4, \\ Heok K Cheow ${ }^{4,5}$, V Krishna Chatterjee ${ }^{1,2}$, Nicholas R Carroll4, Ashley Shaw4, \\ John R Buscombe ${ }^{4,5}$ and Helen L Simpson ${ }^{6}$
}

${ }^{1}$ Metabolic Research Laboratories, Wellcome Trust-MRC Institute of Metabolic Science, University of Cambridge and National Institute for Health Research Cambridge Biomedical Research Centre, Addenbrooke's Hospital, Cambridge, UK

${ }^{2}$ Wolfson Diabetes and Endocrine Centre, Addenbrooke's Hospital, Cambridge, UK

${ }^{3}$ IMED Biotech Unit, Clinical Discovery Unit, AstraZeneca, UK

${ }^{4}$ Department of Radiology, Addenbrooke's Hospital, Cambridge, UK

5Department of Nuclear Medicine, Addenbrooke's Hospital, Cambridge, UK

${ }^{6}$ Department of Diabetes and Endocrinology, UCLH NHS Foundation Trust, London, UK

\begin{abstract}
Objective: In adults with hyperinsulinaemic hypoglycaemia $(\mathrm{HH})$, in particular those with insulinoma, the optimal diagnostic and management strategies remain uncertain. Here, we sought to characterise the biochemical and radiological assessment, and clinical management of adults with $\mathrm{HH}$ at a tertiary centre over a thirteen-year period. Design: Clinical, biochemical, radiological and histological data were reviewed from all confirmed cases of adult-onset hyperinsulinaemic hypoglycaemia at our centre between 2003 and 2016. In a subset of patients with stage I insulinoma, whole-exome sequencing of tumour DNA was performed.

Results: Twenty-nine patients were identified (27 insulinoma, including 6 subjects with metastatic disease; 1 pro-insulin/GLP-1 co-secreting tumour; 1 activating glucokinase mutation). In all cases, hypoglycaemia (glucose $\leq 2.2 \mathrm{mmol} / \mathrm{L}$ ) was achieved within $48 \mathrm{~h}$ of a supervised fast. At fast termination, subjects with stage IV insulinoma had significantly higher insulin, C-peptide and pro-insulin compared to those with insulinoma staged I-IIIB. Preoperative localisation of insulinoma was most successfully achieved with EUS. In two patients with inoperable, metastatic insulinoma, peptide receptor radionuclide therapy (PRRT) with ${ }^{177}$ Lu-DOTATATE rapidly restored euglycaemia and lowered fasting insulin. Finally, in a subset of stage I insulinoma, whole-exome sequencing of tumour DNA identified the pathogenic Ying Yang-1 (YY1) somatic mutation (c.C1115G/p.T372R) in one tumour, with all tumours exhibiting a low somatic mutation burden.

Conclusion: Our study highlights, in particular, the utility of the 48-h fast in the diagnosis of insulinoma, EUS for tumour localisation and the value of PRRT therapy in the treatment of metastatic disease.
\end{abstract}

Correspondence should be addressed to B G Challis Email

bc340@medschl.cam.ac.uk
Endocrine Connections (2017) 6, 540-548

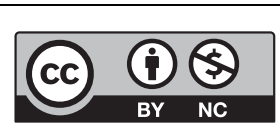

This work is licensed under a Creative Commons Attribution-NonCommercial 4.0 International License. 


\section{Introduction}

Hyperinsulinaemic hypoglycaemia $(\mathrm{HH})$ is uncommon in otherwise healthy adults due to the existence of robust counter-regulatory homeostatic mechanisms that defend against falling blood glucose concentrations. A clinical diagnosis of $\mathrm{HH}$ based on reported symptoms alone is challenging due to their lack of specificity and the attenuation of physiological responses with protracted hypoglycaemia. Whipple's triad states that symptoms compatible with hypoglycaemia occur concomitantly with a low plasma glucose concentration and are ameliorated following restoration of euglycaemia. It is recommended that only once these criteria are satisfied should further investigations be embarked upon to determine the aetiology of the hypoglycaemic disorder (1).

Rare non-genetic and genetic causes of adult-onset $\mathrm{HH}$ have been described, the most common being benign insulinoma. These are insulin-secreting tumours of the pancreatic islets with an annual incidence 1-2 per million population, and associated with multiple endocrine neoplasia (MEN)-1 (1). Although single-copy deletion and somatic mutations in Menin have been identified in some sporadic insulinoma, it is now recognised that a recurrent somatic mutation in the transcription factor Ying Yang $1\left(Y Y 1^{T 372 R}\right)$ occurs in up to one-third of tumours (2). However, for the majority of insulinoma, the underlying genetic event(s) that promote tumourigenesis remain unknown.

Consensus guidelines recommend a supervised fast of up to $72 \mathrm{~h}$ as a practical approach for provoking and establishing a diagnosis of spontaneous hypoglycaemia $(1,3)$. However, given the pressures on inpatient hospital bed capacity and the unpleasantness of a prolonged fast, alternative strategies that are both clinically effective and time-effective warrant consideration. Surgical resection of an insulinoma will be curative for most patients. However, this may be limited by difficulty in successful tumour localisation as the majority of insulinoma are small and often elusive on conventional cross-sectional imaging. Moreover, detection rates for insulinoma with commonly used non-invasive and invasive imaging modalities vary markedly, and currently no consensus exists regarding optimal imaging strategies.

In patients with metastatic disease and intractable hypoglycaemia, maintenance of euglycaemia, progressionfree survival and, importantly, preservation of quality of life form the basis of clinical management. These objectives are not often realised with supplementary carbohydrates alone. Refractory hypoglycaemia may be successfully treated with adjunctive therapeutics including diazoxide, somatostatin analogues or mammalian target of rapamycin (mTOR) inhibitors $(4,5)$. Although these therapies demonstrate clinical efficacy in some patients, intolerable side-effects often render them unsuitable for others. Furthermore, the low incidence of metastatic insulinoma precludes comparison of the clinical efficacy of these therapies in adequately powered, controlled clinical trials. Thus, reporting outcomes from patient series remains a vital conduit for disseminating evidence that additional therapies may be used safely and effectively to manage refractory hypoglycaemia in this rare and clinically challenging patient group.

We present a retrospective analysis of adult patients with biochemically confirmed $\mathrm{HH}$ to characterise their biochemical, radiological assessment and clinical management. Furthermore, we report two cases of inoperable malignant insulinoma with severe refractory hypoglycaemia, in whom PRRT with $\left[{ }^{177} \mathrm{Lu}\right]$-octreotate successfully controlled hypoglycaemia. Finally, in a subset of stage I insulinoma, whole-exome sequencing of tumour DNA was performed, aiming to identify somatic mutations involved in tumour development and autonomous insulin secretion.

\section{Patients and methods}

\section{Study population}

We retrospectively reviewed medical records of patients presenting to our institution between 2003 and 2016 with a diagnosis of hyperinsulinaemic hypoglycaemia, confirmed biochemically during a supervised inpatient fast of up to $72 \mathrm{~h}$. Serum blood glucose was measured every $4-6 \mathrm{~h}$, with a laboratory-assayed plasma glucose of $\leq 2.2 \mathrm{mmol} / \mathrm{L}$ used as the biochemical criterion for fast termination. Notably, in some instances, fasts were terminated despite an absence of neuroglycopenic symptoms, providing the biochemical plasma glucose threshold was achieved. At the time of fast termination, blood was taken for measurement of insulin and, in most cases, pro-insulin and C-peptide. A sulfonylurea screen was also performed. We included patients known to have inherited tumour syndromes, such as MEN-1. We did not include patients with a history of bariatric surgery or with a negative 72-h fast followed by a negative plasma glucose response to intravenous glucagon. We also excluded patients with a

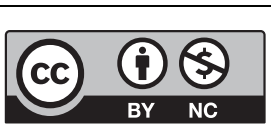

This work is licensed under a Creative Commons Attribution-NonCommercial 4.0 International License. 
negative 72-h fast who exhibited biochemical evidence of reactive hypoglycaemia following either a prolonged (5-h) oral glucose tolerance or mixed-meal test. We collected data related to demographics, clinical history, relevant biochemical and radiological investigations, management, histopathology and follow-up. Tumour staging was reported in accordance with European Tumour Society (ENETS) consensus guidelines (6). Clinical and genetic studies were performed after approval of the National Health Service Research Ethics Committee United Kingdom. Each participant provided written informed consent, and all studies were conducted in accordance with the principles of the Declaration of Helsinki.

\section{Analytic methods}

The assays for measurement of insulin, C-peptide and proinsulin have been previously described (7).

\section{Genomic DNA isolation and whole-exome sequencing}

Genomic DNA extraction from both blood and benign insulinoma samples was performed using the QIAamp DNA Mini Kit (Qiagen) in accordance with manufacturer's instructions. Genomic DNA was quantified using QuantiFluor dsDNA System (Promega) and 50ng of DNA from each sample was used to generate barcoded whole-exome sequencing (WES) libraries according to manufacturer's protocols (Illumina Nextera Rapid Capture Exome Kit, San Diego, CA, USA). WES libraries were quantified by QPCR (KAPA Biosystems, Wilmington, MA, USA) and equal concentrations (10nM) of each library were pooled and subjected to one lane of paired-end 125 bp sequencing on an Illumina HiSeq 2500 System.

\section{Bioinformatics}

WES yielded an average of $19.5 \mathrm{M}$ read pairs per sample and the sequence reads were aligned to the Human UCSC hg38 genome using BWA, version 0.7.5 with an average mapping rate of $99.7 \%$. The aligned reads were then subjected to pre-processing using Picard (version 1.127) and GATK (version 3.30) to correct for duplicate reads, mapping and sequencing artefacts in accordance with GATK best practice guidance (https:// software.broadinstitute.org/gatk/best-practices). Somatic variants were identified using Mutect (version 1.1.7) and annotated using Variant Effect Predictor (version 83, dbNSFP version 3.1a).
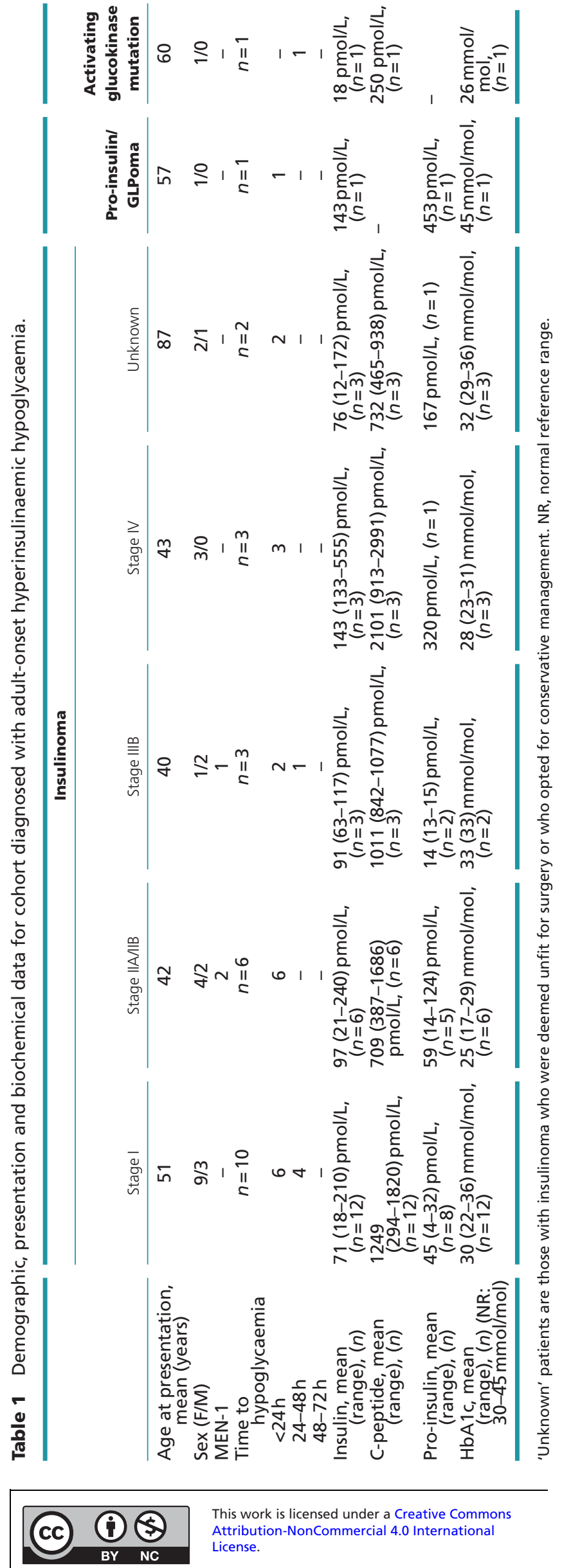

This work is licensed under a Creative Commons Attribution-NonCommercial 4.0 International License. 


\section{Results}

\section{Characteristics of study population}

Twenty-nine patients ( 21 females, 8 males; mean age 52.6, range 16-88 years), with $\mathrm{HH}$ were identified (Table 1). Histopathological analysis confirmed that 27 cases were due to insulinoma (stage I $(n=12)$; stage IIA $(n=5)$; stage IIB $(n=1)$; stage IIIB $(n=3)$; stage IV $(n=3))$. Of these, 3 insulinoma (stage IIA $(n=2)$; stage IIIB $(n=1))$ occurred in the context of MEN-1. We also identified one case of a pro-insulin/GLP-1-secreting tumour and one patient with an activating glucokinase mutation presenting for the first time in adulthood. Details of these two latter cases have been described $(7,8)$. Among the 27 patients with insulinoma, 26 patients reported hypoglycaemic symptoms in the fasting state, whereas 1 patient reported post-prandial symptoms.

\section{Diagnostic testing}

In all patients, a supervised inpatient fast of up to $72 \mathrm{~h}$ was performed to confirm HH. A laboratory-assayed plasma glucose of $\leq 2.2 \mathrm{mmol} / \mathrm{L}$ was the biochemical criterion for fast termination. Of 24 patients with insulinoma for whom fast duration data were available, 19 fasts were terminated within $24 \mathrm{~h}$, and 5 fasts were terminated between 24 and $48 \mathrm{~h}$ (Table 1). Notably, the patient who reported post-prandial symptoms had a positive fast terminated after just seven hours. Among patients with stage IV insulinoma, all fasts were terminated within $24 \mathrm{~h}$. For comparison, and as reported previously, among subjects in our cohort with non-insulinoma-mediated hyperinsulinism, hypoglycaemia was provoked following four hours of fasting in the patient with a pro-insulin/ GLP-1 co-secreting tumour (8), whereas $34 \mathrm{~h}$ of fasting was required to achieve the biochemical criteria for fast termination in the context of an activating GCK mutation (7).

Among patients with insulinoma without distant metastases $(n=24)$, mean insulin concentration (stage I: $71 \mathrm{pmol} / \mathrm{L}$ (range 18-210); stage IIA/IIB: $97 \mathrm{pmol} / \mathrm{L}$ (range 21-240); stage IIIB: 90 pmol/L (range 63-117)) was significantly less than patients with stage 4 disease (405 pmol/L (range 133-555)) $(P<0.001) \quad$ (Fig. 1 and Table 1) at the time of fast termination. Similarly, mean C-peptide concentration was also significantly lower in the absence of distant metastases (stage I: $1249 \mathrm{pmol} / \mathrm{L}$ (range 294-1820); stage IIA/IIB: 709 pmol/L (range 387-1686); stage IIIB: $1011 \mathrm{pmol} / \mathrm{L}$ (range 842-1077)) compared
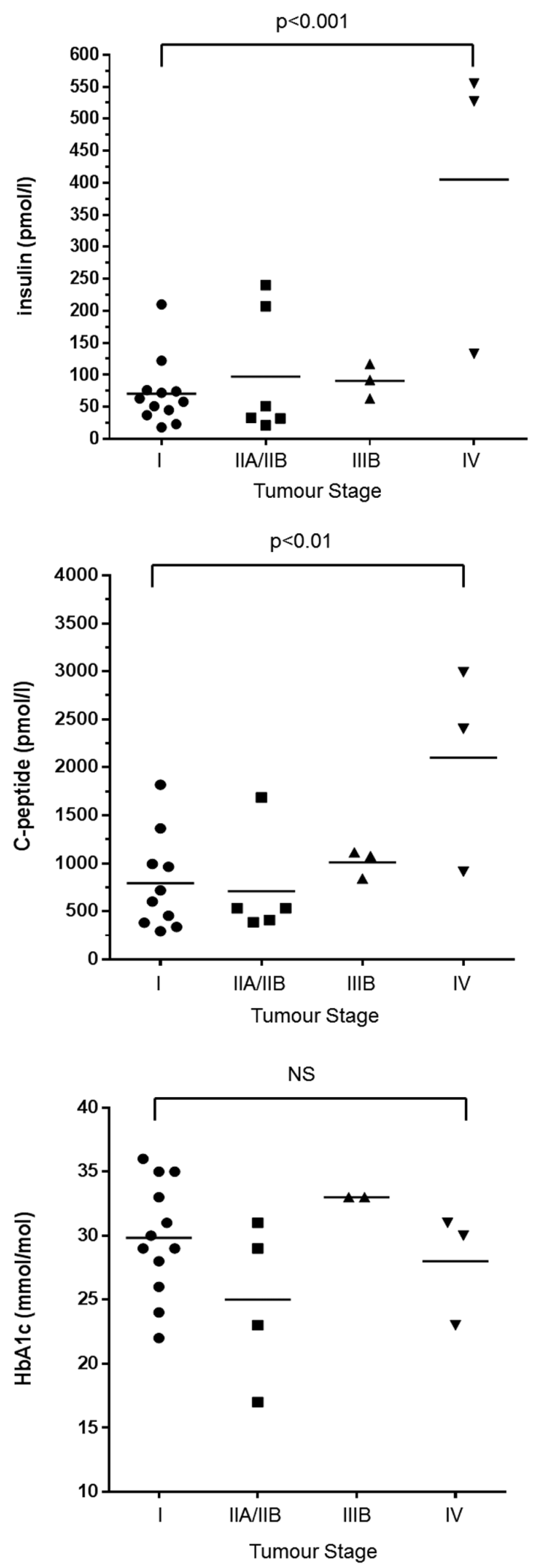

Figure 1

Biochemistry (insulin, C-peptide) at fast termination (plasma glucose $<2.2 \mathrm{mmol} / \mathrm{L}$ ) and presenting $\mathrm{HbA} 1 \mathrm{c}$ in patients with stage I, IIA/IIB, IIIB and IV insulinoma.

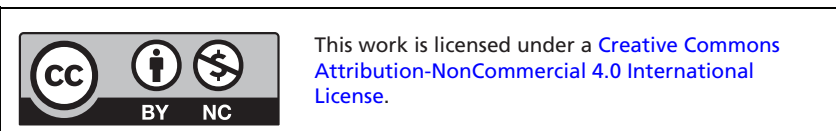


with stage IV disease (2101 pmol/L (range 913-2991)) $(P<0.01)$. There was no statistical difference in HbA1c at presentation between groups (stage 1: $29.8 \mathrm{mmol} / \mathrm{mol}$ (range 22-36); stage IIA/IIB: $25 \mathrm{mmol} / \mathrm{mol}$ (range 17-29); stage IIIB: $33 \mathrm{mmol} / \mathrm{mol}$ ); stage IV: $28 \mathrm{mmol} / \mathrm{mol}$ (range 23-31)). For comparison, similar biochemical parameters are presented for patients with the pro-insulin/GLP-1 co-secreting tumour and activating glucokinase mutation (Fig. 1 and Table 1).

\section{Radiological localisation of insulinoma}

Radiological localisation data are summarised (Fig. 2). Among patients with insulinoma, computed tomography (CT) was the initial imaging modality used in 22 cases. Of these, CT successfully detected the tumour in 15 cases and failed to localise 7 tumours (sensitivity, 68\%). Magnetic resonance imaging (MRI) correctly detected 5 out of 9 insulinoma (sensitivity, 56\%). Of the four tumours not detected, three were also not localised by CT. CT was not performed in the fourth case. Overall, cross-sectional imaging (CT or MRI) detected stage I-IIIB insulinoma in 21 of 24 subjects.

Over the period covered by this retrospective analysis, the preferred invasive preoperative localisation modalities in our centre shifted from pancreatic angiography and intra-arterial calcium stimulation with hepatic vein catheterisation to endoscopic ultrasound (EUS). When used, angiography regionalised 5 of 8 lesions, and when used for a single patient, selective arterial calcium stimulation successfully localised the tumour. Among 22 patients who underwent EUS, insulinoma was correctly

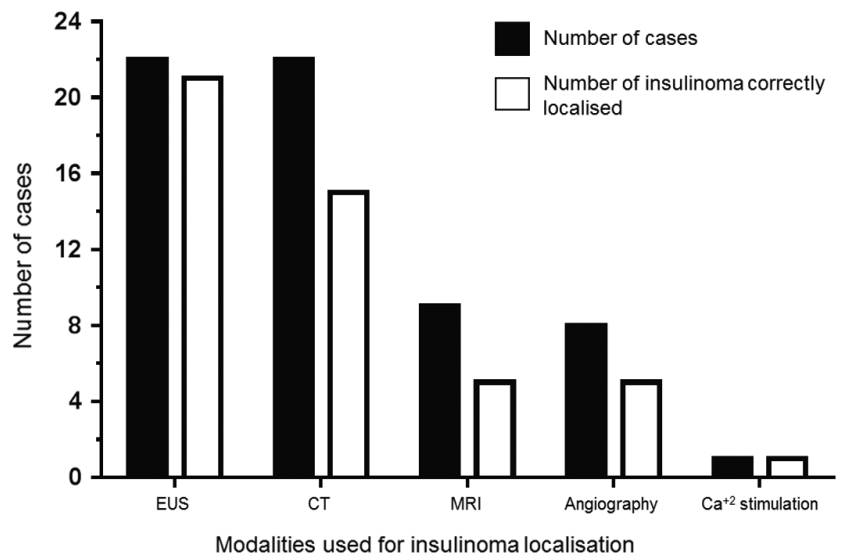

Figure 2

Radiological modalities used to localise benign insulinoma. Black bars indicate number of cases examined with each technique; White bars indicate number of successfully localised insulinoma. detected in 21 cases (sensitivity, 95\%). Moreover, EUS was correctly detected insulinoma in 5 of 6 cases where a tumour was not identified by cross-sectional imaging. Notably, in 3 cases of MEN1-related insulinoma, background microadenomatosis was also visualised by EUS. In the single case where EUS was unsuccessful, calcium stimulation regionalised the tumour to the pancreatic tail.

\section{Clinical management of insulinoma}

Medical management of $\mathrm{HH}$ In addition to regular carbohydrate-based meals and snacks, 28 out of 29 patients required at least one additional supportive therapy to manage hypoglycaemia (individual carbohydrate requirements $1 \mathrm{~g} \mathrm{CHO} / \mathrm{kg}$ every $4-6 \mathrm{~h}$ (9) and 0.2-0.25 g glucose $/ \mathrm{kg} / \mathrm{h}$ to compensate for endogenous glucose production (10)). Dietary complex carbohydrate (corn starch) was the most commonly used adjunct (19 out of 29 patients). Diazoxide was initiated in 14 patients, with dose and scheduling regimes (50 mg once daily to $150 \mathrm{mg}$ three times daily) determined by clinical efficacy and tolerability. Of these, five subjects required concomitant corn starch to maintain euglycaemia. Somatostatin analogue treatment was reserved for patients with octreotide-avid metastatic disease (stage IV insulinoma $(n=3) ; \quad$ metastatic GLP-1/pro-insulin-secreting pNET $(n=1)$. Of these, 1 patient with the GLP-1/pro-insulinsecreting pNET died during the treatment course, whilst a second patient with metastatic insulinoma commenced concomitant systemic chemotherapy (streptozocin and capecitabine). This patient died shortly after treatment initiation. Two patients with malignant insulinoma proceeded to PRRT with $\left[{ }^{177} \mathrm{Lu}\right]$-octreotate following conventional medical management and attempted transarterial embolisation (TAE) of hepatic metastases due to refractory hypoglycaemia (detailed below).

PRRT in patients with malignant insulinoma Case 1: A 32-year-old female presented following severe hypoglycaemia and neuroglycopenic symptoms during labour. HH was confirmed (fasting glucose $0.6 \mathrm{mmol} / \mathrm{L}$, insulin $555 \mathrm{pmol} / \mathrm{L}$, C-peptide $2406 \mathrm{pmol} / \mathrm{L})$. Abdominal CT demonstrated a mass within the pancreatic tail $(83 \mathrm{~mm} \times 53 \mathrm{~mm})$ and multiple hepatic metastases. [ $\left.{ }^{111} \mathrm{In}\right]$-pentetreotide scanning demonstrated avidity throughout the liver, pancreatic tail, left groin, right humeral head and left cervical region (Fig. 3A).

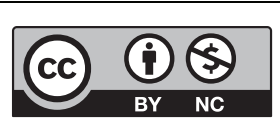

This work is licensed under a Creative Commons Attribution-NonCommercial 4.0 International License. 
A

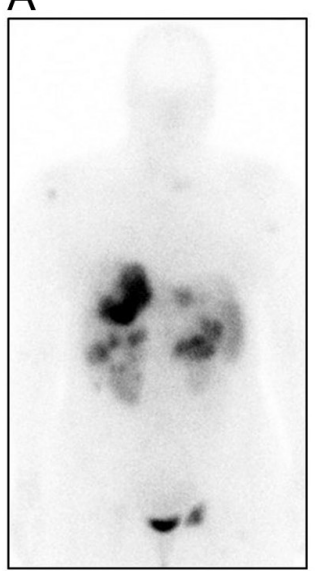

B
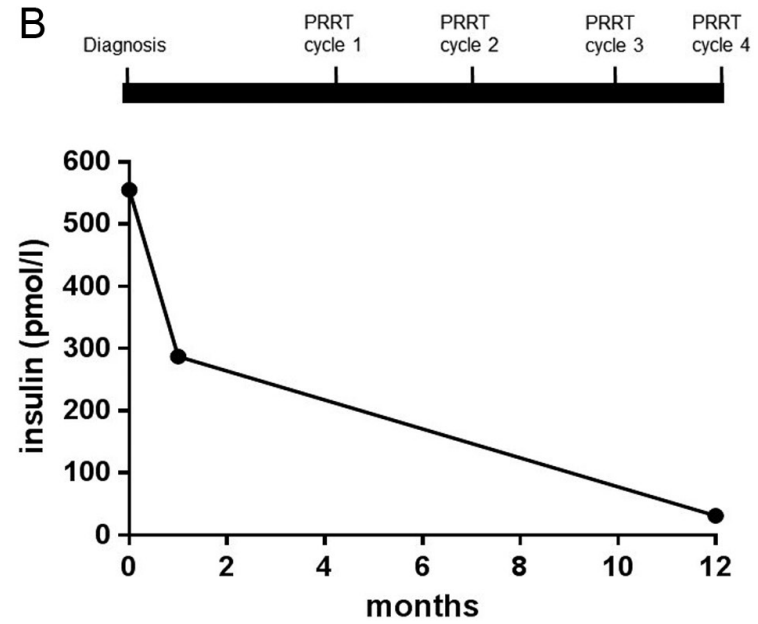

Figure 3

(A) Representative Octreoscan image. (B) Fasting plasma insulin concentrations (pmol/L) in a patient with metastatic (stage IV) insulinoma following treatment with ${ }^{177} \mathrm{Lu}$-Dotatate (PRRT).
Histology of a biopsied liver metastasis revealed a grade 1 well-differentiated pancreatic neuroendocrine tumour (Ki-67 5\%).

Severe hypoglycaemia persisted despite treatment with intravenous 20\% dextrose, corn starch and diazoxide (100 mg tds). Similarly, TAE of hepatic metastases did not control hypoglycaemia. Lanreotide (120 mg every 28 days) was commenced, resulting in less frequent hypoglycaemia. The patient was subsequently treated with four cycles of $\left[{ }^{177} \mathrm{Lu}\right]$-DOTATATE, at 12 -week intervals, up to a total administered activity of $29.6 \mathrm{GBq}$. Hypoglycaemia was immediately abolished following the first cycle of $\left[{ }^{177} \mathrm{Lu}\right]$-DOTATATE. After four cycles, hypoglycaemia remained well controlled, with a significant reduction in peak fasting insulin (31 pmol/L) (Fig. 3B). No renal toxicities were detected; however, pancytopenia developed following the fourth cycle requiring granulocyte-colonystimulating factor and has been slow to improve. To date, 16 months following the first cycle of $\left[{ }^{177} \mathrm{Lu}\right]$-DOTATATE, radiological surveillance demonstrates reduction in size of the primary tumour and some liver metastases, with maintenance of normoglycaemia on normal diet alone.

Case 2: A 32-year-old female presented with slurred speech and confusion associated with minimal exertion and missed meals. During a supervised fast, $\mathrm{HH}$ was confirmed (glucose $1.4 \mathrm{mmol} / \mathrm{L}$, insulin $122 \mathrm{pmol} / \mathrm{L}$, pro-insulin $>200 \mathrm{pmol} / \mathrm{L}$ and C-peptide $1220 \mathrm{pmol} / \mathrm{L})$. Abdominal CT demonstrated a $9 \mathrm{~mm}$ lesion in the pancreatic body and multiple liver metastases. [ $\left.{ }^{111} \mathrm{In}\right]$-pentetreotide scanning failed to demonstrate avidity in the pancreatic mass or liver lesions, whereas ${ }^{68} \mathrm{Ga}$-DOTATE PET-CT revealed moderate avidity in the hepatic lesions and para-aortic lymph nodes. EUS-guided fine needle aspiration of the pancreatic mass, and cytological examination, revealed a well-differentiated grade 1 neuroendocrine tumour (Ki-67-5\%).
Maintenance of euglycaemia required continuous intravenous dextrose (20\%), regular meals (including throughout the night) and corn starch. Diazoxide (100 mg tds) was stopped due to symptomatic tachycardia. TAE of the largest hepatic metastasis failed to reduce hypoglycaemic episodes. $\left.{ }^{[177} \mathrm{Lu}\right]$-DOTATATE treatment was commenced. After just one treatment cycle, hypoglycaemia was abolished and glucose levels remain well controlled with a normal diet and lanreotide (120 mg every 28 days). One month following [ $\left.{ }^{177} \mathrm{Lu}\right]$-DOTATATE, overnight fasting plasma glucose levels were normal (5.5 mmol/L; insulin $59 \mathrm{pmol} / \mathrm{L})$.

Surgical management of insulinoma of 24 patients with suspected localised insulinoma, three either refused surgery or were deemed unfit; these were managed with supportive medical therapies alone. Twenty-one patients underwent surgery, and hypoglycaemia was cured in all cases (laparoscopic distal pancreatecomy; $n=12$; Whipple's procedure; $n=7$; intraoperative enucleation; $n=2$ ).

Follow-up of insulinoma patients Post-operatively, patients with stage I-IIIB insulinoma were assessed clinically and biochemically (HbA1c and/or fasting glucose) at 3, 6 and 12 months, and annually thereafter. Among 20 patients for whom post-operative follow-up data were available, there were no episodes of disease recurrence (median follow-up: 56 months; range: 4-161). Similarly, patients with MEN-1 $(n=3)$ were surveyed in accordance with published consensus guidelines (11), with no evidence of insulinoma recurrence (median follow-up: 75 months; range: 66-150).

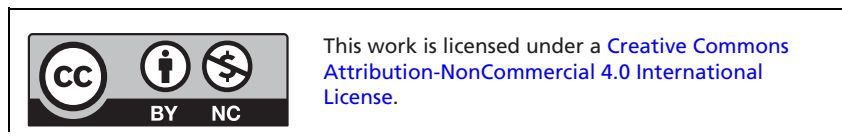




\section{Insulinoma characteristics}

Of the surgically resected insulinoma, 3 tumours were identified within the pancreatic head, 3 in the uncinate process, 8 in the body and 7 in the pancreatic tail. Mean tumour diameter was $15.7 \mathrm{~mm}$ (range 6-45). Sixteen tumours were less than $2 \mathrm{~cm}$ in diameter, 5 less than $2-4 \mathrm{~cm}$ and 1 was greater than $4 \mathrm{~cm}$. In accordance with the ENETS classification (6), tumour staging is shown in Supplementary Table 1 (see section on supplementary data given at the end of this article).

\section{Whole-exome sequencing of benign insulinoma}

Four pairs of DNA extracted from stage I insulinoma and matched blood lymphocytes were selected for WES. The average sequencing depth was 130-fold (111-146), and 97\% (95.6-97.5\%) of the target regions were covered at least 30-fold. Using a coverage cut-off of at least 30-fold, we identified 101 somatic mutations among the 4 tumour exomes (Supplementary Fig. 1), affecting 178 unique genes and 701 coding transcripts. These included 30 synonymous, 61 missense and four nonsense mutations, and 1 splice site mutation within the coding region (Supplementary Fig. 1). Supplementary Table 2 includes somatic mutations predicted to have damaging consequences to gene function, as determined by the in silico prediction tools, SIFT and PolyPhen. The previously reported recurrent somatic mutation in $Y Y 1$ (c.C1115G/p.T372R) was identified in 1 insulinoma and confirmed by Sanger sequencing (data not shown).

\section{Discussion}

In our study, all patients who underwent investigation for hypoglycaemia, and were subsequently diagnosed with insulinoma, had a positive fast within $48 \mathrm{~h}$. This is consistent with other such series, where positive fasts were reported within $48 \mathrm{~h}$ for $>93 \%$ of cases $(3,12)$. Hirshberg and coworkers identified 119 patients with insulinoma; $94.5 \%$ had their fasts terminated within $48 \mathrm{~h}$ when hypoglycaemia (plasma glucose $<2.2 \mathrm{mmol} / \mathrm{L}$ ) was accompanied by neuroglycopenic symptoms (12). Of those in whom fasting was extended beyond $48 \mathrm{~h}$, all had biochemical $\mathrm{HH}$ before $48 \mathrm{~h}$; the fasts were continued because patients did not yet exhibit neuroglycopenic symptoms. In our series, fast termination was based on robust biochemical criteria alone without the requirement for co-existing neuroglycopenic symptoms.
Although discordant with current guidance, and of smaller sample size compared with published studies, our data suggest that a 48 -h fast is effective at identifying the majority of patients who require further investigation, avoiding unnecessarily prolonged fasts in asymptomatic subjects who otherwise exhibit diagnostic biochemistry. Indeed, given that patients with insulinoma may exhibit impaired awareness of hypoglycaemia due to an attenuated sympathoadrenal response following exposure to prolonged antecedent hypoglycaemia, emphasis on the presence of neuroglycopenic symptoms may be unnecessary (13). Notably, however, negative 72-h fasts have rarely been reported in insulinoma patients (14), and clinical judgement remains imperative to avoid misdiagnosis. Similarly, rare causes of non-insulinomamediated hypoglycaemia, such as activating glucokinase mutations, may give rise to a negative 72-h fast (15). In such rare cases, however, a suggestive family history and/or stability of hypoglycaemia throughout a fast are important diagnostic clues.

The small size of most benign insulinoma makes radiological localisation challenging, but this is critical for minimising operating times and improving surgical outcome (16). Conventional non-invasive imaging techniques such as CT or MRI are first-line investigations due to their availability and safety. However, they are inferior to invasive procedures for localising small insulinoma $(16,17)$. Intra-arterial calcium stimulation and EUS have repeatedly been shown to have higher detection rates than non-invasive methods (80-90\% and $75-93 \%$ sensitivity, respectively) $(16,17,18)$. In our series, the detection rate of insulinoma by EUS was 95\% compared with 78\% for CT and MRI. Limitations of EUS include availability, operatorand centre-dependence, invasiveness and reduced ability to detect lesions within the pancreatic tail. Indeed, the single insulinoma in our series not detected by EUS was subsequently regionalised to the pancreatic tail by intraarterial calcium stimulation. An additional benefit of EUS, however, is the ability to undertake biopsies of pancreatic lesions alongside visualisation, thereby confirming diagnosis of a pNET in situ. Moreover, EUS facilitates ablation of pNETs, including insulinoma, for those patients in whom surgery may pose significant risks $(19,20,21)$. Whilst numbers of patients undergoing these procedures remain small, short-term follow-up data and complication rate are encouraging.

Surgical resection with curative intent is standard of care for benign insulinoma. In cases of metastatic disease, refractory hypoglycaemia presents a clinical challenge, contributing to the increased morbidity

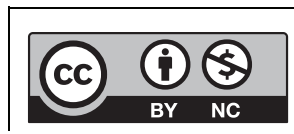

This work is licensed under a Creative Commons Attribution-NonCommercial 4.0 International License. 
and mortality observed for these patients. Therapeutic options for refractory hypoglycaemia include diazoxide, somatostatin analogues, mTOR inhibitors and PRRT. Diazoxide is the most commonly used therapy for refractory hypoglycaemia but has limited efficacy at tolerated doses with recognised adverse effects at higher doses. Similarly, traditional somatostatin analogues (SSA), which demonstrate high affinity for SSTR2 and SSTR5 receptor subtypes, have variable utility, likely reflecting differential expression of somatostatin receptor subtypes among insulinoma (4). Paradoxically, SSA may transiently potentiate hypoglycaemia due to suppression of counterregulatory hormones (22). Abolition of hypoglycaemia and tumour regression has been reported with everolimus $(5,23)$ and this agent is recommended for use in malignant insulinoma resistant to standard medical therapy (24). Adverse effects are common, however, including immunosuppression, myelotoxicity, pneumonitis and cardiac dysfunction (23).

PRRT, using ${ }^{111} \mathrm{In}-,{ }^{177} \mathrm{Lu}$ - or ${ }^{90}$ Yt-labelled somatostatin analogues, is widely used for treatment of metastatic neuroendocrine tumours expressing somatostatin receptors $(4,25)$. In the cases we describe, hypoglycaemia was stabilised within hours of ${ }^{177} \mathrm{Lu}$-octreotate therapy and, in one instance, remains controlled 16 months following PRRT with normal diet and ongoing lanreotide. Other investigators have also reported rapid abolition of hypoglycaemia following PRRT in malignant insulinoma $(4,25)$. Thus, for patients reliant on inpatient support for maintenance of euglycaemia (e.g., with intravenous glucose) PRRT may provide a clinically efficacious option permitting outpatient management and, importantly, improved quality of life. In addition to the metabolic benefits of PRRT, our patients had stable disease radiologically post-PRRT. Whilst our patients were treated only recently, that PRRT has anti-proliferative effects in malignant insulinoma is supported by reports of delayed disease progression of up to 50 months following PRRT (25). Whether PRRT has superior anti-proliferative benefits compared with other currently available systemic therapies, however, remains unclear and given the rarity of malignant insulinoma this question is unlikely to be answered in a sufficiently powered clinical trial.

Through WES of tumour DNA, we and others demonstrate that benign and malignant insulinoma have a low frequency of non-synonymous somatic mutations $(2,26,27)$. This has important clinical relevance, suggesting that immunotherapies targeting checkpoint molecules, such as CTLA- 4 or PD- 1 are unlikely to yield significant clinical benefit in metastatic insulinoma.
Mechanistically, these agents exhibit their anti-tumour effects by enhancing activity of endogenous T cells against cancerous cells and are most clinically efficacious against tumour types with high non-synomymous mutation frequencies and large neoantigen repetoires (28).

For the majority of insulinoma, the molecular events underlying tumour development remain unclear. Indeed, published studies for WES in insulinoma demonstrate minimal overlap between genes containing protein-altering mutations (data not shown). We did not identify somatic mutations in genes previously implicated in development of pancreatic neuroendocrine tumours, including Menin, DAXX or ATRX (29). We did, however, identify a somatic missense mutation in the transcription factor YY1 $\left(Y Y 1^{T 372 R}\right)$ in one benign insulinoma, consistent with previous studies which found $Y Y 1^{T 372 R}$ to occur in up to one-third of such cases (2). Recently, Cromer and coworkers demonstrated that $\mathrm{T} 372 \mathrm{R}$ is a neomorphic mutation that alters the DNA binding specificity of YY1 resulting in marked changes in target gene expression, culminating in autonomous insulin secretion and tumourigenesis (27). Other than the possibility of female preponderance, insulinoma harbouring $Y Y 1^{T 372 R}$ do not appear to differ clinically from wild-type tumours $(26,27)$.

In conclusion, our report adds to the existing literature informing clinical practice for the management of hyperinsulinaemic hypoglycaemia. In particular, our data highlights the validity of a 48-h fast for biochemical diagnosis in suspected insulinoma, and the use of EUS in conjunction with cross-sectional imaging for localisation of insulinoma in order to facilitate curative surgery. Finally, we provide evidence for the utility of PRRT in the management of malignant disease.

\section{Supplementary data}

This is linked to the online version of the paper at http://dx.doi.org/10.1530/ EC-17-0076.

\section{Declaration of interest}

The authors declare that there is no conflict of interest that could be perceived as prejudicing the impartiality of the research reported.

\section{Funding}

A S P is supported by the National Institute for Health Research Cambridge Biomedical Research Centre. R T C is supported by Health Research Board Ireland (Grant reference: HPF-2016-1711). WES was performed at the Genomics and Transcriptomic Core Facility at the Institute of Metabolic Science and the Cambridge Institute Genomics Core, Cancer Research UK. Sequencing and the Human Research Tissue Bank were supported by the NIHR Cambridge Biomedical Research Centre.

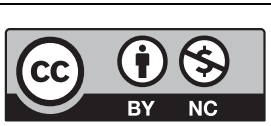




\section{References}

1 Cryer PE, Axelrod L, Grossman AB, Heller SR, Montori VM, Seaquist ER, Service FJ. Evaluation and management of adult hypoglycemic disorders: an Endocrine Society Clinical Practice Guideline. Journal of Clinical Endocrinology and Metabolism 200994 709-728.

2 Cao Y, Gao Z, Li L, Shan A, Cai J, Peng Y, Li Y, Jiang X, Huang X, et al. Whole exome sequencing of insulinoma reveals recurrent T372R mutations in YY1. Nature Communications 201342810

3 Service FJ \& Natt N. The prolonged fast. Journal of Clinical Endocrinology and Metabolism 200085 3973-3974. (doi:10.1210/ jcem.85.11.6934)

4 Ong GS, Henley DE, Hurley D, Turner JH, Claringbold PG \& Fegan PG. Therapies for the medical management of persistent hypoglycaemia in two cases of inoperable malignant insulinoma. European Journal of Endocrinology 2010162 1001-1008. (doi:10.1530/ EJE-09-1010)

5 Kulke MH, Bergsland EK \& Yao JC. Glycemic control in patients with insulinoma treated with everolimus. New England Journal of Medicine 2009360 195-197. (doi:10.1056/NEJMc0806740)

6 Rindi G, Kloppel G, Alhman H, Caplin M, Couvelard A, de Herder WW, Eriksson B, Falchetti A, Falconi M, Komminoth P, et al. TNM staging of foregut (neuro)endocrine tumors: a consensus proposal including a grading system. Virchows Archiv 2006449 395-401. (doi:10.1007/s00428-006-0250-1)

7 Challis BG, Harris J, Sleigh A, Isaac I, Orme SM, Seevaratnam N, Dhatariya K, Simpson HL \& Semple RK. Familial adult onset hyperinsulinism due to an activating glucokinase mutation: implications for pharmacological glucokinase activation. Clinical Endocrinology 201481 855-861. (doi:10.1111/cen.12517)

8 Challis BG, Albrechtsen NJ, Bansiya V, Burling K, Barker P, Hartmann B, Gribble F, O'Rahilly S, Holst JJ \& Simpson HL. Heterogeneity of glucagonomas due to differential processing of proglucagon-derived peptides. Endocrinology, Diabetes and Metabolism Case Reports 20152015 1-7. (doi:10.1530/edm-15-0105)

9 Nyhan WL, Barshop BA \& Ozand PT. Atlas of Metabolic Diseases. 2nd ed., xii 788, pp390. London, UK: Hodder Arnold, 2005.

10 Shaw V \& Lawson M. Clinical Paediatric Dietetics. 3rd ed., ix 620, pp544. Oxford, UK: Blackwell, 2007.

11 Thakker RV, Newey PJ, Walls GV, Bilezikian J, Dralle H, Ebeling PR, Melmed S, Sakurai A, Tonelli F, Brandi ML, et al. Clinical practice guidelines for multiple endocrine neoplasia type 1 (MEN1). Journal of Clinical Endocrinology and Metabolism 201297 2990-3011.

12 Hirshberg B, Livi A, Bartlett DL, Libutti SK, Alexander HR, Doppman JL, Skarulis MC \& Gorden P. Forty-eight-hour fast: the diagnostic test for insulinoma. Journal of Clinical Endocrinology and Metabolism 200085 3222-3226. (doi:10.1210/jc.85.9.3222)

13 Mitrakou A, Fanelli C, Veneman T, Perriello G, Calderone S, Platanisiotis D, Rambotti A, Raptis S, Brunetti P \& Cryer P. Reversibility of unawareness of hypoglycemia in patients with insulinomas. New England Journal of Medicine 1993329 834-839. (doi:10.1056/NEJM199309163291203)

14 Kar P, Price P, Sawers S, Bhattacharya S, Reznek RH \& Grossman AB. Insulinomas may present with normoglycemia after prolonged fasting but glucose-stimulated hypoglycemia. Journal of Clinical Endocrinology and Metabolism 200691 4733-4736. (doi:10.1210/jc.2006-1430)

15 Christesen HB, Brusgaard K, Beck Nielsen H \& Brock Jacobsen B. Noninsulinoma persistent hyperinsulinaemic hypoglycaemia caused by an activating glucokinase mutation: hypoglycaemia unawareness and attacks. Clinical Endocrinology 200868 747-755. (doi:10.1111/j.13652265.2008.03184.x)
16 Guettier JM \& Gorden P. Insulin secretion and insulin-producing tumors. Expert Review of Endocrinology and Metabolism 20105 217-227. (doi:10.1586/eem.09.83)

17 Placzkowski KA, Vella A, Thompson GB, Grant CS, Reading CC, Charboneau JW, Andrews JC, Lloyd RV \& Service FJ. Secular trends in the presentation and management of functioning insulinoma at the Mayo Clinic, 1987-2007. Journal of Clinical Endocrinology and Metabolism 200994 1069-1073. (doi:10.1210/jc.2008-2031)

18 Druce MR, Muthuppalaniappan VM, O'Leary B, Chew SL, Drake WM, Monson JP, Akker SA, Besser M, Sahdev A, Rockall A, et al. Diagnosis and localisation of insulinoma: the value of modern magnetic resonance imaging in conjunction with calcium stimulation catheterisation. European Journal of Endocrinology 2010162 971-978. (doi:10.1530/eje-10-0056)

19 Muscatiello N, Nacchiero M, Della Valle N, Di Terlizzi F, Verderosa G Salcuni A, Macarini L, Cignarelli M, Castriota M, D'Agnessa V, et al. Treatment of a pancreatic endocrine tumor by ethanol injection (PEI) guided by endoscopic ultrasound. Endoscopy 200840 (Supplement 2) E83. (doi:10.1055/s-2007-995540)

20 Pai M, Habib N, Senturk H, Lakhtakia S, Reddy N, Cicinnati VR, Kaba I, Beckebaum S, Drymousis P, Kahaleh M, et al. Endoscopic ultrasound guided radiofrequency ablation, for pancreatic cystic neoplasms and neuroendocrine tumors. World Journal of Gastrointestinal Surgery 20157 52-59. (doi:10.4240/wjgs.v7.i4.52)

21 Waung JA, Todd JF, Keane MG \& Pereira SP. Successful management of a sporadic pancreatic insulinoma by endoscopic ultrasoundguided radiofrequency ablation. Endoscopy 201648 (Supplement 1) E144-E145. (doi:10.1055/s-0042-104650)

22 Stehouwer CD, Lems WF, Fischer HR, Hackeng WH \& Naafs MA. Aggravation of hypoglycemia in insulinoma patients by the long-acting somatostatin analogue octreotide (Sandostatin). Acta Endocrinologica 1989121 34-40. (doi:10.1530/acta.0.1210034)

23 Bernard V, Lombard-Bohas C, Taquet MC, Caroli-Bosc FX, Ruszniewski P, Niccoli P, Guimbaud R, Chougnet CN, Goichot B, Rohmer $\mathrm{V}$, et al. Efficacy of everolimus in patients with metastatic insulinoma and refractory hypoglycemia. European Journal of Endocrinology 2013168 665-674. (doi:10.1530/eje-12-1101)

24 Pavel M, Baudin E, Couvelard A, Krenning E, Oberg K, Steinmuller T, Anlauf M, Wiedenmann B \& Salazar R. ENETS Consensus Guidelines for the management of patients with liver and other distant metastases from neuroendocrine neoplasms of foregut, midgut, hindgut, and unknown primary. Neuroendocrinology 201295 157-176. (doi:10.1159/000335597)

25 van Schaik E, van Vliet EI, Feelders RA, Krenning EP, Khan S, Kamp K, Valkema R, van Nederveen FH, Teunissen JJ, Kwekkeboom DJ, et al. Improved control of severe hypoglycemia in patients with malignant insulinomas by peptide receptor radionuclide therapy. Journal of Clinical Endocrinology and Metabolism 201196 3381-3389. (doi:10.1210/jc.2011-1563)

26 Lichtenauer UD, Di Dalmazi G, Slater EP, Wieland T, Kuebart A, Schmittful A, Schwarzmayr T, Diener S, Wiese D, Thasler WE, et al. Frequency and clinical correlates of somatic Ying Yang 1 mutations in sporadic insulinomas. Journal of Clinical Endocrinology and Metabolism 2015100 E776-E782. (doi:10.1210/jc.2015-1100)

27 Cromer MK, Choi M, Nelson-Williams C, Fonseca AL, Kuntsman JW, Korah RM, Overton JD, Mane S, Kenney B, Malchoff CD, et al. Neomorphic effects of recurrent somatic mutations in Yin Yang 1 in insulin-producing adenomas. PNAS 2015112 4062-4067. (doi:10.1073/pnas.1503696112)

28 Schumacher TN \& Schreiber RD. Neoantigens in cancer immunotherapy. Science 2015348 69-74.

29 Jiao Y, Shi C, Edil BH, de Wilde RF, Klimstra DS, Maitra A, Schulick RD, Tang LH, Wolfgang CL, Choti MA, et al. DAXX/ATRX, MEN1, and mTOR pathway genes are frequently altered in pancreatic neuroendocrine tumors. Science 2011331 1199-1203.

Received in final form 3 July 2017

Accepted 7 August 2017

Accepted Preprint published online 7 August 2017 http://www.endocrineconnections.org DOI: 10.1530/EC-17-0076
(C) 2017 The authors Published by Bioscientifica Ltd
This work is licensed under a Creative Commons Attribution-NonCommercial 4.0 International License. 